Voix et Images

voixetimages

\title{
Le poème comme recueil, tableau, espace
}

\section{André Brochu}

Volume 36, numéro 3 (108), printemps-été 2011

URI : https://id.erudit.org/iderudit/1005128ar

DOI : https://doi.org/10.7202/1005128ar

Aller au sommaire du numéro

Éditeur(s)

Université du Québec à Montréal

ISSN

0318-9201 (imprimé)

1705-933X (numérique)

Découvrir la revue

Citer ce compte rendu

Brochu, A. (2011). Compte rendu de [Le poème comme recueil, tableau, espace].

Voix et Images, 36(3), 126-131. https://doi.org/10.7202/1005128ar d'utilisation que vous pouvez consulter en ligne.

https://apropos.erudit.org/fr/usagers/politique-dutilisation/ 


\author{
P O É S I E \\ Le poème commerecueil, tableau, espace \\ $+++$ \\ ANDRÉ BROCHU \\ Université de Montréal
}

On constate aujourd'hui une tendance à interroger le poème non plus seulement tel qu'en lui-même, en ses thèmes et ses procédés formels (ses thèmes surtout), mais aussi en rapport avec sa structure "éditoriale» (le recueil) ou, encore, dans ses correspondances avec les arts voisins, notamment la peinture.

\title{
LE RECUEIL COMME ÉCRITURE
}

Dans un petit livre intitulé Le poème en recueil ${ }^{1}$, François Dumont propose une amorce de réflexion sur les rapports entre le poème et l'espace-livre où il prend place. Quelle est l'incidence du dispositif éditorial sur la lecture du texte, pourtant considéré à bon droit comme autonome ${ }^{2}$ ? Sujet intéressant, mais difficile. Il permet à l'auteur d'effectuer de fines analyses, mais on n'est pas sûr qu'il ait été pleinement traité.

En effet, les six chapitres du livre étaient à l'origine autant d'études, sans lien entre elles, publiées dans des revues à plusieurs années d'intervalle, et on y cherche en vain la trace d'une problématique en bonne et due forme concernant une poétique du recueil. Les deux pages de l'«Introduction» ne suffisent pas à l'esquisser. Elles indiquent une direction de recherche, sans plus : «L'intention du présent ouvrage est de considérer les diverses dimensions de ce contexte éditorial qui est aussi une forme d'écriture et qui joue un rôle important dans la lecture.» (5) À cette fin, quelques œuvres marquantes de notre poésie sont abordées : les Poésies complètes d'Hector de Saint-Denys Garneau, L'homme rapaillé de Gaston Miron, Poèmes des quatre côtés de Jacques Brault, les trois «rétrospectives » de Roland Giguère et Le sens du soleil de Pierre Nepveu. On aimerait avoir au moins quelques indications sur d'autres recueils phares de nos lettres, comme Les îles de la nuit d'Alain Grandbois, Le tombeau des rois d’Anne Hébert, Mémoire sans jours de Rina Lasnier, Le réel absolu de Paul-Marie

$$
+++
$$

1 François Dumont, Le poème en recueil, Québec, Nota bene, 2010, 152 p. 2 On peut citer, dans une semblable perspective, l'ouvrage de Catherine Morency, L'atelier de L'âge de la parole: poétique du recueil chez Roland Giguère, Montréal, Les heures bleues, coll. «Le dire», 2006, 128 p. 
Lapointe...

Le dernier chapitre aborde la question de l'anthologie poétique. Celle-ci est l'objet d'une étude formelle dont on aurait souhaité trouver l'équivalent à propos des recueils qui précèdent. Il y a là les bases d'une typologie qui fait appel à des choix qu'on pourrait qualifier d'idéologiques: on privilégie la poésie (sans référence au contexte littéraire national) ou, au contraire, la nation; la chronologie ou l'ordre alphabétique des auteurs; la sélection des textes ou celle des auteurs, etc.

Que serait une typologie des recueils? On peut imaginer, en gros, une échelle d'ouvrages qui irait du plus libre (indiscipliné) au plus construit. En domaine français, je pense à Alcools d'Apollinaire où se succèdent des poèmes en vers libres («Zone») et des poèmes en vers réguliers («Le pont Mirabeau», «La chanson du mal-aimé») avec une belle désinvolture. Les autres recueils du poète, même les Calligrammes - et malgré la présence de poèmes-dessins (les calligrammes, justement) - , répondent eux aussi à un souci d'hétérogénéité formelle. Voilà bien la preuve que le recueil peut n'obéir qu'à des exigences minimums. À l'inverse, la Délie de Maurice Scève est composée uniquement de dizains (il y en a 449), formés de décasyllabes. Voilà donc un objet littéraire d'une grande homogénéité, tant formelle que thématique. Le paradis des apparences, de Robert Melançon, avec ses 144 poèmes de douze vers, lui fait écho en notre siècle. On peut imaginer aussi une orchestration rigoureuse de la diversité, par exemple Regards et jeux dans l'espace de Saint-Denys Garneau, dont les sections, très différentes les unes des autres, nous transportent de façon progressive de l'expression initiale du bonheur et de l'enfance à celle du malheur et de la mort, suivie d'un bref retour à la lumière. On observe une démarche semblable dans Le tombeau des rois d'Anne Hébert.

François Dumont évite les théorisations rapides, comme celle que je viens de commettre, et fait plutôt œuvre de critique attentif à la singularité des œuvres. Il aborde les recueils sans s'attarder longuement à leur carapace formelle, mais décrit tout de même celle-ci (et reproduit les tables des matières en appendice) et la met tout de suite en relation avec la signification des poèmes. Ses analyses sont souvent fondées sur celles de prédécesseurs, tel Jacques Blais ou Michel Biron. Le titre de chaque chapitre renvoie simultanément aux dispositions architecturales du texte et aux thèmes qui leur sont liés, par exemple «L'atelier du rassemblement» pour les nombreuses versions de L’homme rapaillé, «Une poétique pluraliste» pour la rétrospective de Pierre Nepveu, etc.

Bref, on pourrait souhaiter une approche plus rigoureuse, mais ce livre suggestif ouvre plus d'horizons que ne le ferait une visée bien systématique.

\section{POÉSIE ET PEINTURE}

Il n'est pas trop tard pour parler d'une étude considérable et fort intéressante parue il y a deux ans, L'école du regard, d'Antoine Boisclair ${ }^{3}$. Certes, ce qui fut à l'origine une thèse

$$
++
$$

3 Antoine Boisclair, L'école du regard. Poésie et peinture chez Saint-Denys Garneau, Roland Giguère et Robert Melançon, Montréal, Fides, coll. « Nouvelles études québécoises», 2009, 430 p. 
de doctorat mériterait plus qu'une page ou deux, et cela, dans une chronique plus savante que la présente, mais la parution vaut le coup de chapeau. Elle porte sur trois poètes québécois de haut niveau, qui appartiennent à des générations successives. Saint-Denys Garneau est l'homme de La Relève, revue des années 1930 où s'exprimait un catholicisme progressiste qui prépara les voies de la Révolution tranquille. Roland Giguère est de la génération des automatistes et des surréalistes québécois qui se manifesta peu de temps après la dernière guerre mondiale (le mythique Refus global parut en 1948). Enfin, Robert Melançon est de cette génération dite des baby-boomers qui chercha sa voie dans le formalisme puis dans une expression lyrique de la subjectivité - Melançon, toutefois, fut le tenant d'une forme rigoureuse de réalisme (le sous-titre du Paradis des apparences ${ }^{4}$ se lit: «essai de poèmes réalistes »), qui conjugue la représentation exacte des choses au souci formel de l'expression. De Saint-Denys Garneau à Robert Melançon, on suit donc l'évolution des rapports entre poésie et image depuis le chant de l'existence, d'inspiration spirituelle, à l'affirmation du surréel qui évacue la religion tout en cultivant la voyance, puis à une attention exacte au réel qui n'est peut-être pas étrangère à une modernité marquée par la technologie et l'approche mathématique de la nature (petit exemple: «Le soleil photographie les arbres,/En noir et blanc, sur le trottoir ${ }^{5}$ »).

Quoi qu'il en soit de la «vision » propre à chacun des auteurs, ce qui les réunit est une même attention accordée à l'aspect visuel, "pictural» des choses, ce qui va d'ailleurs faire de Saint-Denys Garneau et de Roland Giguère des praticiens de la peinture et, de Melançon, un peintre à sa façon (verbale, descriptive), sans parler de leur commune ferveur en tant qu'amateurs d'art. Notons, bien entendu, que ces trois tenants de l'Ut pictura poesis (" un poème est comme un tableau»), pour reprendre la formule d'Horace, ne sont pas les seuls à s'affirmer comme tels dans notre tradition poétique, mais sans doute sont-ils les plus représentatifs. Antoine Boisclair fait cependant un recensement minutieux des prédécesseurs et des accompagnateurs, d'Eudore Évanturel à Jacques Brault, tout comme il fait état des nombreuses théories qui fondent la problématique des rapports entre peinture et poésie tout au long de l'histoire occidentale, de Zeuxis d'Héraclée ou de Simonide à Yves Bonnefoy ${ }^{6}$.

Il est passionnant de voir comment une égale attention esthétique à la représentation visuelle est valorisée de façon fort différente selon les poètes, alors que d'autres poètes seront plutôt fascinés par un art comme la musique. La poétique de chacun s'en ressent, d'une façon qu'on a encore peu étudiée.

Trois formules, selon Antoine Boisclair, résument la démarche des auteurs à l'étude : apprendre à voir (Saint-Denys Garneau), donner à voir (Roland Giguère) et faire voir (Robert Melançon), ce qui montre bien que le regard peut être engagé dans des stratégies significatives très personnelles.

4 Robert Melançon, Le paradis des apparences. Essai de poèmes réalistes, Montréal, Éditions du Noroît, 2004, 150 p. (144 pages, selon la pagination personnelle de l'auteur dont les poèmes, de douze vers chacun, sont au nombre de $12 \times 12=144$ ). 5 Ibid., p. 51.6 Il faudrait aussi mentionner toute une tradition du livre d'artiste, dont ceux - parmi tant d'autres - de Lucie Lambert auxquels rend hommage un collectif sous la direction de Réjean Beaudoin, L'essence scripturale du trait gravé (Montréal, Éditions du Noroît, coll. "Chemins de traverse», 2010, 80 p.). On y trouve notamment des témoignages d'Yvon Rivard, de Jacques Brault, de Robert Melançon, de Jean-Marc Fréchette et de Jean-Pierre Duquette. 
Pour l'auteur de Regards et jeux dans l'espace, le regard est comme une lame doublement affûtée qui, pour bien se frayer un chemin à l'extérieur, doit s'enfoncer tout aussi profondément à l'intérieur (133-134). Jusqu'en 1937, le poète s'adonne avec ferveur aux exercices de vision, générateurs de communication et facteurs d'harmonie, lesquels alimentent sa peinture et son inspiration littéraire. Il entretient «un rapport généralement franc et heureux avec le visible» (138). Ici, on peut se demander pourquoi les poèmes de Garneau souvent considérés comme ses meilleurs sont ceux de la fin, où il a pris ses distances à l'égard du visible et s'est enfermé dans le noir de la solitude et du désespoir. La réponse serait-elle dans son poème intitulé «Faction", où une "petite étoile problématique ${ }^{7}$ » doit sa visibilité à la nuit qu'on a faite sur la terre? Il n'en reste pas moins qu'Antoine Boisclair semble considérer les «Esquisses en plein air ", où la poésie se fait peinture (aquarelle) de la façon la plus convaincante (149-150), comme la réussite par excellence de Saint-Denys Garneau, ce qui peut sembler discutable. On conclurait plutôt que la valeur du poème ne tient pas vraiment à l'usage que le poète a fait de la dimension visuelle puisque celle-ci est absente de ses plus grandes réussites.

Curieusement, malgré la différence des problématiques garnélienne et giguérienne, une ressemblance troublante s'affirme entre les œuvres sur le plan de la trajectoire parcourue. Roland Giguère aboutit, comme Saint-Denys Garneau, à une «suite de tableaux et de poèmes intitulés Pouvoir du noir (1966), où le motif de l'infigurable acquiert une importance accrue» (225). Pour l'un et l'autre poète, le figural (ou descriptif) est dépassé en un dicible sans image, la poésie récuse la peinture. L'interaction peinture-poésie est un point de départ, non un point d'arrivée. Toutefois, on ne peut pas affirmer que le meilleur de la poésie de Giguère corresponde à sa période «noire » (finale), contrairement à ce qui se passait pour Saint-Denys Garneau. Le lien entre le voir et le dire, la peinture et la poésie, est trop immédiatement décisif chez Giguère pour n'avoir pas déterminé de grandes réussites, tant littéraires qu'artistiques.

Après des aperçus substantiels sur Robert Marteau, Fernand Ouellette et Jacques Brault, diversement marqués par l'attrait du voir se donnant pour objet l'«invu» ou, même, l'invisible, Antoine Boisclair aborde l'œuvre de Robert Melançon, particulièrement riche en références à la peinture. Le poète est tourné de façon fort décidée vers le fini, qu'il s'agit de faire voir avec la plus grande précision possible, dégagé de tout enveloppement de voyance (contrairement à ce que "donne à voir » Roland Giguère). Tout en soulignant le réalisme très poussé de Melançon, Antoine Boisclair montre chez lui l'importance de la figure géométrique (372), qui le rapproche du cubisme en peinture. L'absence de lyrisme se trouverait alors compensée par le sortilège de l'abstraction mathématique, tant il est vrai que la poésie, si elle peut rejeter la voyance, ne saurait se passer de quelque magie. Faire voir le réel, qui est du reste une illusion, un trompe-l'œil, c'est le faire voir le plus réel possible sans escamoter - ce serait impossible - ce contre quoi il s'affirme et qui fait aussi partie de lui: sensibilité, ou intelligibilité, ou spiritualité... Chez Melançon, le réel

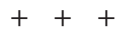

7 Saint-Denys Garneau, «Faction", Regards et jeux dans l'espace, Euvres, édition de Jacques Brault et Benoît Lacroix, Montréal, Presses de l'Université de Montréal, 1971, p. 27. 
est intelligible, dénué de sentiments immédiats, fin, correct et contenu en tranches de douze vers.

Le beau livre d'Antoine Boisclair nous fait découvrir tout un aspect de la poésie québécoise jusqu'ici négligé au profit de préoccupations identitaires. Ces dernières sont certes importantes, mais il faut savoir les mettre en perspective avec d'autres. Voilà l'apport majeur de ce livre à la fois ambitieux et nuancé.

\section{GILLES CYR ET L'ESPACE}

Il y a une vingtaine d'années, les théoriciens de la poésie n'en avaient que pour le temps. Maintenant, c'est l'espace qui retient toute l'attention. On l'a vu dans l'ouvrage consacré à «l'école du regard», qui aborde la poésie en relation avec l'art pictural. La chose est plus claire encore dans le non moins savant essai de Marc André Brouillette, Spatialité textuelle dans la poésie contemporaine ${ }^{8}$, auquel je me contenterai de renvoyer. Le tiers du livre est consacré à la poésie de Gilles Cyr, qui fait justement paraître une réédition en un volume de ses quatre premiers recueils ${ }^{9}$, avec une préface du même Marc André Brouillette.

Je parlerai donc des poèmes, qui sont connus et qui ont fait l'objet d'appréciations fort élogieuses de la part des amateurs et des spécialistes. Marc André Brouillette, dans sa préface, réunit toutes les indications qui permettent au lecteur non initié de se familiariser avec la poésie de Gilles Cyr. Voilà qui est très utile car Gilles Cyr, comme Robert Melançon, a construit son œuvre à l'écart des tendances de la poésie québécoise. Dès le début, il propose un ton et un style rigoureusement personnels, qui ne se démentiront pas par la suite, mais qui déconcertent le lecteur habitué à des discours plus coutumiers. Je pense à des titres de recueil comme Diminution d'une pièce ou Andromède attendra. Ces énoncés énigmatiques, que n'éclairent aucunement les poèmes qu'ils chapeautent, ne cachent pas une vérité (par exemple, d'ordre mythologique) qu'il s'agirait de découvrir par une démarche de connaissance ou un effort de réflexion; ils gardent leur épaisseur d'énigme dans la mesure même où ils disent, à leur façon, l'impénétrabilité irréductible du réel. Diminution d'une pièce: de quelle "pièce »? S'agit-il d'un morceau appartenant à un ensemble fonctionnel? Ou de la division d'une habitation? Et cette Andromède, est-ce bien la fille de Cassiopée que Poséidon, irrité contre sa mère, attache à un rocher où elle attend d'être dévorée? Son histoire, en tout cas, n'est nullement reprise dans la suite des poèmes.

Pourtant ceux-ci, de facture très semblable, reposent sur une ferme isotopie. Il y est question de la terre (du sol), du ciel, des montagnes, de tout ce qui compose à grands traits l'espace où nous vivons. Il y est question des démarches très simples qu'accomplit le sujet («je») dans cet espace. Ce sujet n'a rien d'un moi lyrique, d'une intériorité rêveuse. Il existe en symbiose immédiate avec le monde qui nous est décrit.

$$
+++
$$

8 Marc André Brouillette, Spatialité textuelle dans la poésie contemporaine. Gilles Cyr, Jean Laude et Anne-Marie Albiach, Québec, Nota bene, 2010, 298 p. 9 Gilles Cyr, Poèmes 1968-1994, Montréal, TYPO, 2010, 320 p. 
Il s'agit bien du monde, car nous sommes aux antipodes d'une poésie du pays ou d'une thématique de l'enracinement. Ici, l'universel est appréhendé à partir du détail concret de la nature et du rapport qu'il appelle avec le sujet. Rapport souvent déconcertant puisque l'expérimentation incessante du monde est traduite de façon fort elliptique et progresse de métonymie en métonymie, jamais au gré de métaphores. À preuve ce poème cité par le préfacier comme exemple de saugrenu (légitime):

\author{
Tu es là \\ j'ai une minute \\ il se rapproche \\ le scélérat \\ tu as bonne mine \\ Armand! \\ je ne sais pas \\ si je peux y aller \\ tiens prends ça \\ qu'est-ce que l'art? \\ si tu ne veux pas \\ je veux te revoir (270).
}

Le langage très quotidien est comme troué pour faire apparaître les éléments d'un drame (ou d'une comédie ?) dont on n'a pas la clé et qui ressemble sans doute à nos vies dans ce qu'elles ont à la fois de simple et d'indéchiffrable. 